\title{
Arranjo de Estímulos em Treino Discriminativo Simples com Compostos e Emergência de Classes de Estímulos Equivalentes
}

\author{
Márcio Borges Moreira ${ }^{1}$ \\ Centro Universitário de Brasília, Brasília, DF, Brasil \\ Adriana Oliveira \\ Universidade de Brasilia, Brasília, DF, Brasil \\ Elenice S. Hanna \\ Departamento de Processos Psicológicos Básicos da Universidade de Brasília, \\ Brasilia, DF, Brasil
}

\section{Resumo}

O treino discriminativo simples simultâneo com compostos (DSSi) tem produzido variabilidade nos resultados de testes de estímulos equivalentes. O presente trabalho investigou em dois estudos o papel de variáveis metodológicas, que alteram as chances de controle por aspectos não definidos como relevantes, sobre a formação de classes de estímulos equivalentes. O Estudo 1 avaliou o efeito da utilização de um composto incorreto (S-) para cada composto correto (S+) em cada tentativa do DSSi que garantia o contexto de discriminação condicional sem redundância (e.g., A1B1 com A1B2 ou A1B1 com A2B1). Cinco estudantes universitários aprenderam as discriminações básicas e mostraram relações simétricas e transitivas. O Estudo 2 verificou o efeito da inclusão de um elemento redundante (mancha preta) nos compostos de uma das classes, utilizando um delineamento intra-sujeito. Avaliou-se o efeito dessa manipulação em um procedimento de treino de relações condicionais arbitrárias com DSSi sobre os desempenhos nos testes de relações emergentes, utilizando-se MTS e DSSi. Os doze universitários mostraram relações simétricas, mas apenas cinco apresentaram relações transitivas. Esses resultados expandem as evidências de que configurações das tentativas de treino e as características irrelevantes dos estímulos podem gerar falso positivo nos treinos discriminativos.

Palavras-chave: Discriminações simples simultâneas, estímulos compostos, relações arbitrárias emergentes, estudantes universitários.

1 Endereço para correspondência: Universidade de Brasília, Instituto de Psicologia, Departamento de Processos Psicológicos Básicos, Campus Universitário Darcy Ribeiro, ICC Norte, Asa Norte, Caixa-postal: 4500, Brasilia, DF, Brasil 70910-900. E-mail: borgesmoreira@gmail.com, oliveiradeadriana@gmail.com e hanna@unb.br O estudo é parte da tese de doutorado do primeiro autor e da dissertação de mestrado da segunda autora realizadas no Programa de Pós-Graduação em Ciências do Comportamento da Universidade de Brasília, sob a orientação da terceira autora. Adriana Oliveira contou com bolsa da Coordenação de Aperfeiçoamento de Pessoal de Nível Superior (CAPES) para realização do mestrado. Os autores são membros do Instituto Nacional de Ciência e Tecnologia sobre Comportamento, Cognição e Ensino, financiado pelo Conselho Nacional de Desenvolvimento Científico e Tecnológico - CNPq (Processo \#573972/2008-7) e pela Fundação de Amparo à Pesquisa do Estado de São Paulo - FAPESP (Processo \# 2008/57705-8), que apoiou o desenvolvimento dos estudos. 


\title{
Stimulus Arrangement in Simple Discriminative Training with Compounds and Emergence of Stimulus Equivalence Clases
}

\begin{abstract}
The simple simultaneous discrimination training with compounds (SSD) have produced variability in the results of equivalent stimuli tests. This study investigated in two studies the role of methodological variables that alter the chances of control by aspects not defined as relevant on the formation of stimulus equivalence classes. Study 1 evaluated the effect of using an incorrect compound (S-) for each correct compound $(\mathrm{S}+)$ in each SSD trial that granted the conditional discrimination context without redundancy (eg, A1B1 with A1B2 or A1B1 with A2B1). Five college students learned the basic discriminations and showed symmetrical and transitive relations. The second study assessed the effect of the inclusion of a redundant element (a black spot) in the compounds of one of the classes using a within-subject design. The effect of this manipulation in a training procedure of arbitrary conditional relations with SSI on performances in emerging relation tests, using MTS and SSI was evaluated. The twelve students showed symmetrical relations, but only five showed transitive relations. These results expand the evidence that training trial settings and irrelevant characteristics of the stimuli may generate false positives in discriminative training.
\end{abstract}

Keywords: Simple simultaneous discrimination, compound stimuli, arbitrary emergent relations, college students.

\section{Arreglo de Estímulo en Compuestos Simples con Entrenamiento Discriminativo y la Clase Equivalente de Estímulos}

\section{Resumen}

El entrenamiento discriminativo sencilla simultáneamente con compuestos (DSSi) han producido variabilidad en los resultados de las pruebas de estímulos equivalentes. Este estudio investigó en dos estudios el papel de las variables metodológicas que alteran las posibilidades de control por parte de los aspectos no definidos como relevantes, sobre la formación de las clases de equivalencia de estímulo. Estudio 1 evaluó el efecto del uso de un compuesto incorrecta $(\mathrm{S}+)$ para cada compuesto correcta $(\mathrm{S}+)$ en cada intento de garantía DSSi que el contexto discriminación condicional sin redundancia (por ejemplo, A1B1 con A1B2 A1B1 o con A2B1). Cinco estudiantes universitarios aprendieron la discriminación básica y mostraron relaciones simétricas y transitivas. El segundo estudio evaluó el efecto de la inclusión de un elemento redundante (punto negro) en los compuestos de las clases utilizando un diseño intrasujeto. Se evaluó el efecto de esta manipulación en un procedimiento de formación de las relaciones condicionales arbitrarias con DSSi sobre actuaciones en relaciones emergentes pruebas, utilizando MTS y DSSi. Los doce estudiantes mostraron relaciones simétricas, pero sólo cinco tenido relaciones transitivas. Estos resultados amplían la evidencia de que los intentos de formación configuraciones y características irrelevantes de los estímulos pueden generar falsos positivos en la práctica discriminatoria.

Palabras clave: Discriminación simples concurrentes, compuestos de incentivos, emergentes relaciones arbitrarias, universidad estudiantes.

O termo estímulo composto tem sido usado em contextos de pesquisa experimental para se referir à apresentação conjunta, justaposição ou sobreposição daquilo que se convencionou chamar de estímulos unitários (e.g., Grisante et al.,
2013; Markham \& Dougher, 1993; Ray, 1969; Reynolds, 1961). Resultados experimentais têm, entretanto, demonstrado que partes ou propriedades de estímulos considerados unitários podem exercer controle sobre o comportamento 
(e.g., Lashley, 1938; Touchette, 1969), sugerindo que essa distinção entre estímulo composto e unitário é arbitrária.

Uma série de estudos tem analisado empírica e conceitualmente o paradigma de equivalência (Sidman et al., 1982; Sidman \& Tailby, 1982) a partir da noção de que elementos de um estímulo composto podem ser separados e recombinados sem que haja disruptura no controle discriminativo (cf., Stromer, McIlvane, \& Serna, 1993). Markham e Dougher (1993) e Stromer e Stromer (1990), por exemplo, demonstraram a formação de classes de equivalência de estímulos utilizando um procedimento de pareamento ao modelo (matching to sample, MTS) no qual os estímulos utilizados como modelo eram estímulos compostos (um som e uma cor) e os estímulos de comparação eram estímulos unitários (figuras). Durante os testes de simetria e transitividade esses estímulos foram decompostos e apresentados separadamente, ora como modelo e ora como comparação para avaliar as relações condicionais estabelecidas (ver também, Carpentier, Smeets, \& Barnes-Holmes, 2000; Groskreutz, Karsina, Miguel, \& Groskreutz, 2010; Guerrero, Alós, \& Moriana, 2015; Maguire, Stromer, Mackay, \& Demis, 1994; Pérez-González \& Alonso-Álvarez, 2008; Ribeiro, Miguel, \& Goyos, 2015).

A formação de classes de equivalência de estímulos também foi demonstrada em estudos que utilizaram estímulos compostos em treinos de discriminações simples simultâneas (DSSi; e.g., Moreira \& Hanna, 2012; Smeets, Barnes-Holmes, \& Cullinan, 2000) e de discriminações simples sucessivas (DSSu; e.g., Debert, Huziwara, Faggiani, de Mathis, \& McIlvane, 2009; Debert, Matos, \& McIlvane, 2007). Em ambos os casos, com participantes adultos, foi demonstrada a emergência das relações de simetria, de transitividade e de simetria da transitividade. Os testes de relações emergentes com procedimentos de discriminação simples (DSSi e DSSu) são realizados com a recombinação dos elementos dos estímulos composto (e.g., se o composto A1B1 foi estabelecido com S+, B1A1 é utilizado no teste de simetria).
Pesquisas que avaliam a formação de classes de equivalência geralmente utilizam procedimentos semelhantes para treino e teste. Por exemplo, se o treino foi realizado com MTS, os testes de emergência de novas relações também são realizados utilizando-se o procedimento de MTS. Moreira e Hanna (2012) parecem ter sido os primeiros a realizarem os testes com um procedimento diferente daquele utilizado durante os treinos. Os autores realizaram dois estudos nos quais os participantes foram submetidos a treinos de DSSi com estímulos compostos. Em ambos os estudos, o desempenho emergente dos participantes foi testado tanto com um procedimento de treino de DSSi quanto com MTS.

No Estudo 1 de Moreira e Hanna (2012), nove estudantes de graduação foram submetidos ao treino de duas discriminações $\mathrm{AB}$ e duas discriminações $\mathrm{BC}$ utilizando-se um estímulo composto correto $(\mathrm{S}+)$ e dois estímulos compostos incorretos (S-) em cada tentativa. Estímulos compostos corretos eram aqueles formados por componentes pertencentes ao mesmo conjunto definido pelo experimentador (e.g., A1B1), e estímulos compostos incorretos eram aqueles formados por componentes pertencentes a conjuntos diferentes (e.g., A1B2). Os $\mathrm{S}$ - foram os mesmos em todas as tentativas de treino de cada relação (e.g., A1B2 e B2A1, presentes em todas as tentativas do Treino $\mathrm{AB}$ ). Todos os participantes do Estudo 1 atingiram o critério de aprendizagem estabelecido para os treinos $(100 \%$ de acerto no último bloco de tentativas de cada treino), apresentando poucos ou nenhum erro durante os demais blocos de tentativas e mostraram emergência das relações simétricas testadas. No entanto, com relação aos testes de transitividade e simetria da transitividade, diferentemente de Smeets et al. (2000, para os participantes adultos), por exemplo, observou-se padrões do tipo "tudo-ou-nada" ( $100 \%$ de acerto ou $0 \%$ de acerto) intra e entre-sujeitos, sugerindo que a formação de classes a partir de procedimentos de DSSi com compostos, da forma como foi realizado o estudo, necessita ser melhor investigada. Os percentuais de acerto para todas as relações fo- 
ram semelhantes em ambos os procedimentos de teste utilizados (MTS e DSSi).

De acordo com Moreira e Hanna (2012), a variabilidade dos resultados relativa à emergência das relações de transitividade e simetria da transitividade do Estudo 1, entre e intra-sujeitos, pode ter ocorrido, pelo menos em parte, em função do número reduzido de S- (apenas dois), implicando a repetição dos mesmos dois $\mathrm{S}-$ em todas as tentativas de treino, aumentando as chances de estabelecimento de controle por rejeição dos $\mathrm{S}$ - ou outro controle espúrio permitido pela combinação dos estímulos que compunham cada tentativa de treino. Por exemplo, em uma tentativa com os compostos A1B1(S+), A1B2(S-) e A2B1(S-), em vez do comportamento do participante ficar sob o controle da relação entre os elementos do composto $\mathrm{S}+$, esse poderia ser controlado por qualquer composto que combina os elementos mais frequentes de cada tentativa (e.g., na tentativa com A1B1, A1B2 e $\mathrm{A} 2 \mathrm{~B} 1, \mathrm{~A} 1 \mathrm{~B} 1$ é escolhido porque tanto A1 quanto $B 1$ aparecem duas vezes). Não fica claro no estudo de Moreira e Hanna porque os possíveis controles espúrios teriam comprometido apenas a emergência das relações de transitividade e simetria da transitividade.

No Estudo 2 de Moreira e Hanna (2012), seguindo a mesma estrutura de treinos e testes do Estudo 1, cinco estudantes de graduação foram submetidos ao treino de três discriminações $\mathrm{AB}$ e três discriminações $\mathrm{BC}$ (em relação a duas discriminações $\mathrm{AB}$ e duas $\mathrm{BC}$ estabelecidas no Estudo 1) e o par de estímulos compostos apresentados como S- em cada tentativa variou entre as tentativas de treino e de teste em função da inserção do terceiro conjunto de estímulos, o que possibilitou o aumento de combinações possíveis de $\mathrm{S}$ - de dois para seis (A1B2, A1B3, A2B1, A2B3, A3B1 e A3B2, para o treino AB e $\mathrm{B} 1 \mathrm{C} 2, \mathrm{~B} 1 \mathrm{C} 3, \mathrm{~B} 2 \mathrm{C} 1, \mathrm{~B} 2 \mathrm{C} 3, \mathrm{~B} 2 \mathrm{C} 1$ e $\mathrm{B} 3 \mathrm{C} 2$ para o Treino BC). Todos os participantes aprenderam as relações ensinadas, apresentando poucos ou nenhum erro e demonstraram a emergência de todas as relações testadas. Assim como no Estudo 1 , não houve diferença no desempenho dos participantes em função do tipo de procedimento de teste utilizado (MTS ou DSSi).
Os estudos de Moreira e Hanna (2012) demonstraram a emergência de relações condicionais arbitrárias utilizando dois procedimentos de teste diferentes para os mesmos participantes e relações, mas não foi possível identificar com precisão a variável relevante para explicar a variabilidade encontrada no Estudo 1. A inserção de um terceiro conjunto de estímulos no Estudo 2, além de possibilitar um maior número de S-, aumentou, em relação ao Estudo 1, o número mínimo de tentativas de treino programadas de 152 para 234. Essa maior exposição às contingências de reforço programadas também pode ter favorecido a aprendizagem das relações de controle de estímulos programadas pelo experimentador, implicando melhores desempenhos nos testes de transitividade e simetria da transitividade.

No presente trabalho, com base em questões levantadas por Moreira e Hanna (2012), dois estudos procuraram evidências adicionais sobre as variáveis importantes para a formação de classes de estímulos equivalentes a partir do ensino de discriminações simples com estímulos compostos. O Estudo 1 avaliou o efeito da configuração das tentativas de treino, reduzindo o número de S- presentes em cada tentativa. Para o Estudo 2, criou-se explicitamente um aspecto espúrio (independente dos elementos dos compostos) em um dos S+ para avaliar a formação de classes de estímulos equivalentes.

\section{Estudo 1}

Dois estudos que utilizaram treino de DSSi com dois estímulos compostos relataram resultados divergentes. Smeets et al. (2000) mostraram emergência de todas as relações testadas com adultos, enquanto que Moreira e Hanna (2012, Estudo 1) observaram variabilidade na emergência das relações de transitividade e simetria da transitividade. A fim de adicionar informações sobre fatores que geraram esta divergência nos resultados, o primeiro estudo do presente trabalho replicou o Estudo 1 de Moreira e Hanna, reduzindo o número de $\mathrm{S}$ - programado em cada tentativa de dois para um. Esta modificação permitiu que os S- de cada conjunto fossem alter- 
nados entre tentativas e tornou o procedimento mais semelhante ao de Smeets et al..

\section{Método}

\section{Participantes}

Participaram do Estudo 1 cinco universitários, um do sexo masculino e quatro do sexo feminino, com idades variando entre 22 e 57 anos. Foram concedidos aos participantes créditos de atividade complementar pela participação no estudo (crédito de uma hora para cada sessão realizada). Todos os participantes assinaram o Termo de Consentimento Livre e Esclarecido e o projeto foi aprovado pelo Comitê de Ética em Pesquisa com Seres Humanos do Instituto de Ciências Humanas da Universidade de Brasília.

\section{Ambiente Experimental, Equipamento e Estímulos}

As sessões foram realizadas em cabines experimentais medindo aproximadamente $2 \times 2 \mathrm{~m}$ com isolamento acústico. Em cada cabine havia uma mesa, uma cadeira e um micro-computa- dor com mouse, teclado, monitor (de 15 ou 17 polegadas) e ambiente operacional Windows $\mathrm{XP}{ }^{\circledR}$ e browser Internet Explorer ${ }^{\circledR}$ (versão 6.0 ou superior). A tarefa experimental e o registro dos dados foram feitos utilizando-se o software MTS_DSSint 1.0 (Moreira \& Hanna, 2012).

Os estímulos utilizados foram figuras abstratas de baixa nomeabilidade extraídas do trabalho de Nalini (2002). As figuras mediam aproximadamente $2 \times 2 \mathrm{~cm}$ e foram elaboradas na cor preta sob um fundo branco. Estímulos utilizados para um treinamento inicial da tarefa foram selecionados a partir do banco de autoformas do Microsoft PowerPoint $\AA$, também na cor preta sob fundo branco e com dimensões similares às das demais figuras utilizadas. A Figura 1 apresenta os elementos que compuseram os estímulos utilizados e dois exemplos de estímulos compostos. Os estímulos eram apresentados, na tela do computador, dentro de retângulos brancos, medindo $5,5 \mathrm{~cm}$ de largura por $4 \mathrm{~cm}$ de altura. Um estímulo composto (e.g., A1B1) era formado pela apresentação, lado a lado dentro do retângulo branco, dos estímulos unitários (e.g., A1 e B1), conforme exemplificado na Figura 1.

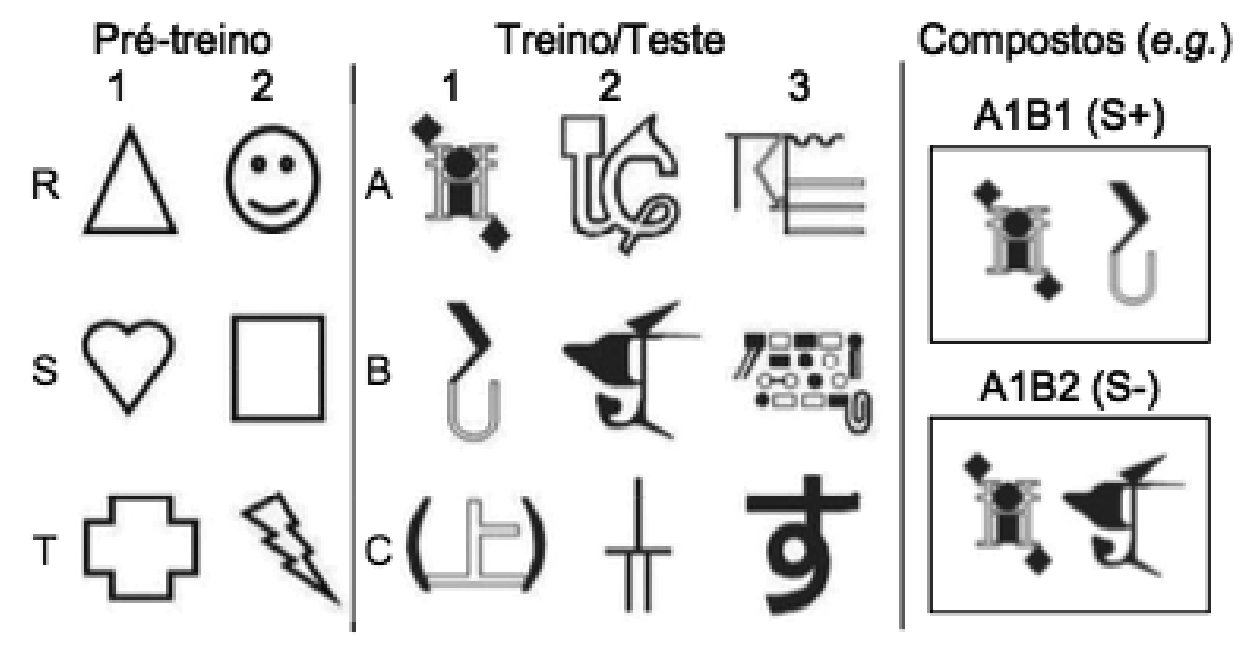

Figura 1. Elementos e exemplos de compostos utilizados como estímulos nos Estudos 1 e 2. Cada número (1, 2 e 3) designa um conjunto de estímulos e cada letra corresponde a um exemplar do conjunto. O Conjunto 3 foi utilizado apenas no Estudo 2. O Elemento A do Conjunto 1, por exemplo, é descrito no texto como A1.

\section{Procedimento}

Cada participante foi submetido a três protocolos de sessão experimental, que programavam treinos de DSSi seguidos por testes DSSi e MTS em, no mínimo, três sessões com dura- ção aproximada de 20 minutos. $\mathrm{O}$ termo protocolo refere-se neste trabalho a uma sequência específica de procedimentos de treino e testes de discriminações que compunham uma sessão experimental. 
O Protocolo 1 era composto por Pré-Treino, Treino AB, Teste BA, Teste A-B ${ }^{2}$ e Teste B-A. O Protocolo 2 era composto por Treino $\mathrm{BC}$, Teste CB, Teste B-C e Teste C-B. O Protocolo 3 era composto por Treino Misto (relações AB e BC), Teste AC, Teste A-C, Teste CA e Teste C-A. A Tabela 1 apresenta as configurações dos treinos e testes realizados em cada sessão e na ordem em que foram realizados. Os Testes BA, CB, $\mathrm{AC}$ e $\mathrm{CA}$ foram realizados com procedimento de DSSi e os Testes A-B, B-A, B-C, C-B, A-C e C-A com MTS.

Pré-Treino. Cada participante foi inicialmente submetido a um pré-treino para familiarização com a tarefa experimental com os estímulos apresentados no painel esquerdo da Figura 1. O participante era instruído a observar as figuras e clicar sobre uma delas. Era informado ao participante que se a sua resposta estivesse correta, apareceria a palavra "Certo" na tela. O Pré-treino constituiu-se de 13 blocos com número de tentativas e critérios semelhantes aos dos treinos (descritos abaixo), exceto que não havia repetição de blocos quando erros eram cometidos. Parte do Pré-treino (os cinco blocos finais) foi realizada com procedimento de MTS com estímulos unitários (similar ao procedimento de teste com MTS descrito a seguir, mas com a adição de uma consequência diferencial - a mesma descrita para os procedimentos de treino).

DSSi

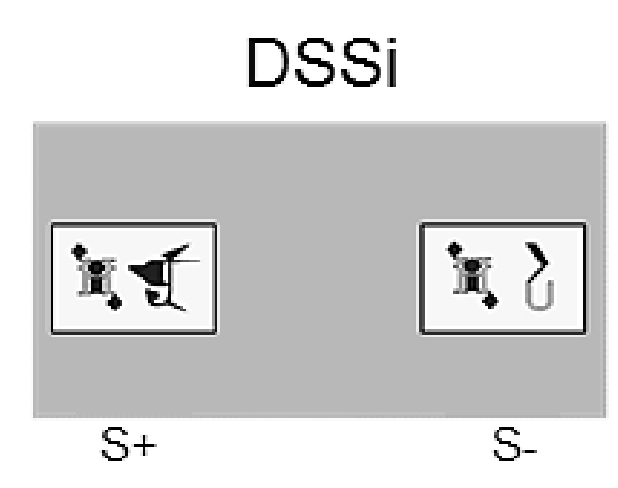

Treinos. Os treinos foram realizados com um procedimento de treino de discriminação simples simultânea (DSSi) com estímulos compostos (Figura 2). Cada tentativa de treino iniciava-se com a apresentação na tela do computador de um ou dois estímulos compostos sobre fundo azul-claro. Quando dois estímulos compostos eram apresentados, um tinha a função de $\mathrm{S}+\mathrm{e}$ ou outro de S- (Tabela 1). Os estímulos compostos eram apresentados dentro de retângulos brancos dispostos horizontalmente, lado a lado, centralizados verticalmente, aqui chamado de janela de resposta. Ao posicionar-se o ponteiro do mouse dentro dos limites de uma janela, uma borda de cerca de $2 \mathrm{~mm}$ na cor azul-escuro aparecia ao redor desta, e desaparecia quando o cursor era movimentado para fora da janela ou quando fosse efetuado um clique sobre ela. Respostas de clicar sobre a janela do estímulo composto positivo $(\mathrm{S}+)$ produziam a retirada dos estímulos da tela e a apresentação da palavra "CERTO" por $1,5 \mathrm{~s}$. Após esse tempo, iniciava o intervalo entre tentativas (IET) com duração de $2,3 \mathrm{~s}$, durante os quais a tela permanecia na cor marrom. Ao final do IET uma nova tentativa se iniciava. Se a resposta ocorresse no composto negativo (S-), a tentativa finalizava e iniciava o IET com a tela marrom. Imediatamente após o IET, a tentativa na qual houve erro era apresentada novamente com os mesmos estímulos nas mesmas posições.

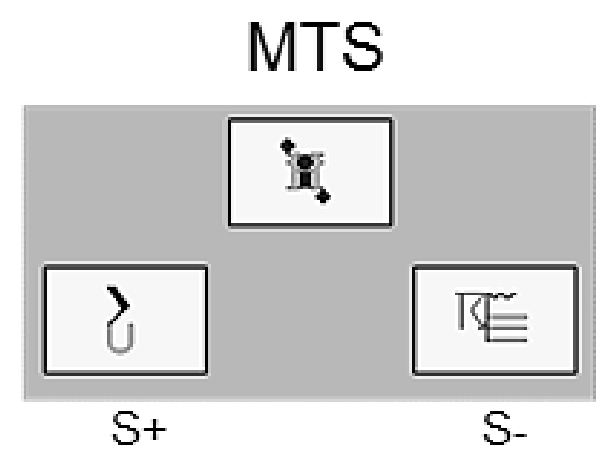

Figura 2. Configuração da tela de apresentação de estímulos de uma tentativa dos procedimentos DSSi e MTS.

Procedimentos de treino ou teste com MTS são indicados com letras separadas por hífen (e.g., A-B) e aqueles com DSSi são descritos com letras unidas (e.g., AB).
A ordem de apresentação dos $\mathrm{S}+$, bem como a posição destes e dos S- na tela (esquerda, centro ou direita) foi semi-randomizada e um mesmo $\mathrm{S}+$ não era apresentado por mais de 3 tentativas 
consecutivas, nem na mesma posição (a mesma ordem de apresentação das tentativas por bloco foi repetida para todos os participantes).

Os treinos foram organizados em blocos de tentativas com o aumento gradual do número de estímulos presentes em cada tentativa e do número de $\mathrm{S}+$ apresentados nas tentativas de cada bloco (Tabela 1). Em todos os blocos de treino, um ou mais erros produziam a repetição do bloco de tentativas com a ordem de apresentação alterada. Erros consecutivos em três blocos finalizavam a sessão, que era repetida em outro dia a partir do início do protocolo, incluindo o Pré-teste no caso do Protocolo 1. Portanto, para finalizar os treinos de um protocolo o participante precisava obter $100 \%$ de acerto em todos os blocos.

Treino $A B$ e $B C$. Os treinos $\mathrm{AB}$ e $\mathrm{BC}$ foram programados com a mesma estrutura, alterando-se apenas as relações treinadas (A1B1 e A2B2 no Treino $\mathrm{AB}$ e $\mathrm{B} 1 \mathrm{C} 1$ e $\mathrm{B} 2 \mathrm{C} 2$ no Treino $\mathrm{BC}$ ). $\mathrm{Na}$ primeira tentativa de cada treino, o primeiro $\mathrm{S}+$ era apresentado isoladamente e nos três blocos seguintes ( 2 a 4, Tabela 1 ) um S- era apresentado com o $\mathrm{S}+$. Em seguida, a mesma sequência era realizada com o segundo $\mathrm{S}+$ (blocos $5 \mathrm{a}$ 7). Nos últimos dois blocos (9 e 10) todos os $\mathrm{S}+$ e S- eram alternados entre tentativas (Tabela 1).

Treino Misto. No Treino Misto, cada S+ era apresentado sozinho uma vez (Bloco 1, Tabela 1). Em seguida era programado um bloco de tentativas com as discriminações $\mathrm{AB}$ (Bloco 2), um bloco com as discriminações BC (Bloco 3) e três blocos misturando tentativas com as discriminações $\mathrm{AB}$ e $\mathrm{BC}$, todos com o $\mathrm{S}+\mathrm{e}$ um $\mathrm{S}-$ (blocos 4,5 e 6). O Bloco 6 foi programado em extinção (i.e., nenhuma consequência foi planejada para respostas corretas e incorretas). Antes de iniciar este treino o participante era avisado, na tela do computador, que não mais seria informado se estava acertando.

Testes BA, $C B, A C$ e $C A$. Os Testes BA, CB, $\mathrm{AC}$ e $\mathrm{CA}$ foram realizados utilizando-se um procedimento de DSSi com estímulos compostos (Figura 2), como nos treinos. Cada teste constituiu-se de um bloco com seis tentativas (ver Tabela 1), sendo três apresentações de cada relação testada (B1A1, B2A2, C1B1, C2B2, A1C1, $\mathrm{A} 2 \mathrm{C} 2, \mathrm{C} 1 \mathrm{~A} 1$ e C2A2). Os estímulos compostos utilizados nos testes com DSSi foram recombinações dos estímulos compostos utilizados nos treinos, alterando-se a posição relativa (esquerda ou direita) de que cada elemento do estímulo composto (e.g., B1A1, A1C1, C2A2). Em cada tentativa, respostas de clicar sobre qualquer um dos estímulos produziam a retirada dos mesmos da tela (nenhuma consequência diferencial foi programada para acerto e erro). Após a retirada dos estímulos, iniciava o IET com duração de $2,3 \mathrm{~s}$, durante os quais a tela permanecia na cor marrom.

Testes $A-B, B-A, B-C, C-B, A-C$ e $C-A$. Os Testes A-B, B-A, B-C, C-B, A-C e C-A foram realizados utilizando-se um procedimento de MTS com estímulos unitários (Figura 2), sempre com duas escolhas. Para os testes com MTS, os estímulos compostos utilizados nos treinos foram apresentados separadamente (e.g., B1 apresentado como modelo e A1 apresentado como comparação; Tabela 1). Cada teste constituiu-se de um bloco com seis tentativas (Tabela 1), sendo três apresentações de cada relação testada (A1-B1, A2-B2, B1-A1, B2-A2, B1-C1, B2-C2, C1-B1, C2-B2, A1-C1, A2-C2, C1-A1 e C2-A2).

Nos testes com MTS cada tentativa iniciavase com a apresentação de um estímulo modelo, centralizado no alto da tela azul claro (e.g., B1). A resposta de clicar sobre o estímulo modelo produzia a apresentação imediata e simultânea de dois estímulos de comparação, sendo um positivo e um negativo (e.g., A1 e A2). Em cada tentativa, respostas de clicar sobre qualquer um dos estímulos de comparação produziam a retirada dos mesmos da tela. Após a retirada dos estímulos, iniciava o IET com duração de $2,3 \mathrm{~s}$, durante os quais a tela permanecia na cor marrom.

Caso o participante, nos Testes BA, A-B, B-A, CB, B-C e C-B, clicasse sobre um S-, em mais de uma tentativa de uma mesma relação (e.g., tentativas nas quais B1A1 era $\mathrm{S}+$ ), estes testes eram repetidos após o Treino Misto, antes dos testes programados para o Protocolo 3. Para todos os testes, utilizou-se o critério de aprendizagem de $83,33 \%$ para se dizer que houve emergência das relações testadas. 
Tabela 1

Protocolos das Tentativas de Treino e Teste de cada Sessão Experimental, com Especificação dos Estímulos Positivos (S+) e Negativos (S-) e Número de Tentativas (NT) Programado em cada Bloco (Bl)

\begin{tabular}{|c|c|c|c|c|c|c|c|c|c|c|c|}
\hline \multicolumn{4}{|c|}{ Protocolo 1} & \multicolumn{4}{|c|}{ Protocolo 2} & \multicolumn{4}{|c|}{ Protocolo 3} \\
\hline $\mathrm{Bl}$ & $\mathrm{S}+$ & S- & NT & $\mathrm{Bl}$ & $\mathrm{S}+$ & S- & NT & $\mathrm{Bl}$ & $\mathrm{S}+$ & S- & NT \\
\hline \multicolumn{4}{|c|}{ Treino AB } & \multicolumn{4}{|c|}{ Treino BC } & \multicolumn{4}{|c|}{ Treino Misto $\mathrm{AB} / \mathrm{BC}$} \\
\hline 1 & A1B1 & - & 1 & 1 & $\mathrm{~B} 1 \mathrm{C} 1$ & - & 1 & 1 & A1B1 & - & 4 \\
\hline 2 & A1B1 & A1B2 & 4 & 2 & $\mathrm{~B} 1 \mathrm{C} 1$ & $\mathrm{~B} 1 \mathrm{C} 2$ & 4 & & A2B2 & - & \\
\hline 3 & A1B1 & A2B1 & 4 & 3 & $\mathrm{~B} 1 \mathrm{C} 1$ & $\mathrm{~B} 2 \mathrm{C} 1$ & 4 & & $\mathrm{~B} 1 \mathrm{C} 1$ & - & \\
\hline \multirow[t]{2}{*}{4} & A1B1 & A1B2 & 6 & 4 & $\mathrm{~B} 1 \mathrm{C} 1$ & $\mathrm{~B} 1 \mathrm{C} 2$ & 6 & \multirow{3}{*}{2} & $\mathrm{~B} 2 \mathrm{C} 2$ & - & \\
\hline & & A2B1 & & & & $\mathrm{B} 2 \mathrm{C} 1$ & & & A1B1 & A1B2 & 8 \\
\hline 5 & $\mathrm{~A} 2 \mathrm{~B} 2$ & - & 1 & 5 & $\mathrm{~B} 2 \mathrm{C} 2$ & - & 1 & & A2B2 & A2B1 & \\
\hline 6 & A2B2 & A1B2 & 4 & 6 & $\mathrm{~B} 2 \mathrm{C} 2$ & $\mathrm{~B} 1 \mathrm{C} 2$ & 4 & 3 & $\mathrm{~B} 1 \mathrm{C} 1$ & $\mathrm{~B} 1 \mathrm{C} 2$ & 8 \\
\hline 7 & A2B2 & A2B1 & 4 & 7 & $\mathrm{~B} 2 \mathrm{C} 2$ & $\mathrm{~B} 2 \mathrm{C} 1$ & 4 & \multirow{5}{*}{4} & $\mathrm{~B} 2 \mathrm{C} 2$ & $\mathrm{~B} 2 \mathrm{C} 1$ & \\
\hline \multirow[t]{2}{*}{8} & \multirow[t]{2}{*}{ A2B2 } & A1B2 & \multirow[t]{2}{*}{6} & \multirow[t]{2}{*}{8} & \multirow[t]{2}{*}{ B2C2 } & $\mathrm{B} 1 \mathrm{C} 2$ & \multirow[t]{2}{*}{6} & & A1B1 & A1B2 & \multirow[t]{2}{*}{8} \\
\hline & & A2B1 & & & & $\mathrm{B} 2 \mathrm{C} 1$ & & & A2B2 & A2B1 & \\
\hline \multirow[t]{2}{*}{9} & A1B1 & A1B2 & \multirow[t]{2}{*}{8} & \multirow[t]{2}{*}{9} & $\mathrm{~B} 1 \mathrm{C} 1$ & $\mathrm{~B} 1 \mathrm{C} 2$ & \multirow[t]{2}{*}{8} & & $\mathrm{~B} 1 \mathrm{C} 1$ & B1C2 & \\
\hline & A2B2 & A2B1 & & & $\mathrm{B} 2 \mathrm{C} 2$ & $\mathrm{~B} 2 \mathrm{C} 1$ & & & $\mathrm{~B} 2 \mathrm{C} 2$ & $\mathrm{~B} 2 \mathrm{C} 1$ & \\
\hline \multirow[t]{3}{*}{10} & A1B1 & A1B2 & \multirow[t]{3}{*}{12} & 10 & $\mathrm{~B} 1 \mathrm{C} 1$ & $\mathrm{~B} 1 \mathrm{C} 2$ & \multirow[t]{2}{*}{12} & \multirow[t]{4}{*}{5 e $6^{*}$} & A1B1 & A1B2 & \multirow[t]{4}{*}{12} \\
\hline & $\mathrm{A} 2 \mathrm{~B} 2$ & A2B1 & & & $\mathrm{B} 2 \mathrm{C} 2$ & $\mathrm{~B} 2 \mathrm{C} 1$ & & & A2B2 & A2B1 & \\
\hline & \multicolumn{2}{|c|}{ Teste BA/B-A } & & \multicolumn{4}{|c|}{ Teste CB/C-B } & & $\mathrm{B} 1 \mathrm{C} 1$ & $\mathrm{~B} 1 \mathrm{C} 2$ & \\
\hline 1 & $\mathrm{~B} 1 \mathrm{~A} 1$ & $\mathrm{~B} 2 \mathrm{~A} 1$ & 6 & 1 & C1B1 & $\mathrm{C} 1 \mathrm{~B} 2$ & 6 & & $\mathrm{~B} 2 \mathrm{C} 2$ & $\mathrm{~B} 2 \mathrm{C} 1$ & \\
\hline & $\mathrm{B} 2 \mathrm{~A} 1$ & $\mathrm{~B} 1 \mathrm{~A} 2$ & & & C2B2 & C2B1 & & & te $\mathrm{AC} / \mathrm{A}$ & $\mathrm{C} / \mathrm{CA} / \mathrm{C}-$ & \\
\hline 2 & A1 & B1 & 6 & 2 & B1 & $\mathrm{C} 1$ & 6 & 1 & $\mathrm{AlC1}$ & $\mathrm{A} 1 \mathrm{C} 2$ & 6 \\
\hline & $\mathrm{A} 2$ & B2 & & & B2 & $\mathrm{C} 2$ & & & $\mathrm{~A} 2 \mathrm{C} 2$ & $\mathrm{~A} 2 \mathrm{C} 1$ & \\
\hline 3 & B1 & A1 & 6 & 3 & $\mathrm{C} 1$ & B1 & 6 & 2 & A1 & $\mathrm{C} 1$ & 6 \\
\hline & B2 & $\mathrm{A} 2$ & & & $\mathrm{C} 2$ & B2 & & & A2 & $\mathrm{C} 2$ & \\
\hline & & & & & & & & 3 & C1A1 & $\mathrm{C} 1 \mathrm{~A} 2$ & 6 \\
\hline & & & & & & & & & $\mathrm{C} 2 \mathrm{~A} 2$ & $\mathrm{C} 2 \mathrm{~A} 1$ & \\
\hline & & & & & & & & 4 & $\mathrm{C} 1$ & A1 & 6 \\
\hline & & & & & & & & & $\mathrm{C} 2$ & A2 & \\
\hline
\end{tabular}

Nota. Todas as tentativas apresentavam no máximo dois estímulos, um positivo e um negativo; as tentativas com estímulos unitários foram testes com o procedimento de pareamento ao modelo. *O Bloco 6 do Treino Misto (Protocolo 3 ) foi idêntico ao Bloco 5, exceto pela ausência de consequências diferencias.

\section{Resultados}

As porcentagens de acertos nos treinos e testes são apresentadas na Figura 3. As primeiras três colunas de cada gráfico, na cor branca, mostram os percentuais de acerto de cada participante no último bloco de tentativas de cada treino. Todos os participantes atingiram 100\% de acerto no último bloco dos Treinos AB, BC e Misto.

Nos testes, todos os participantes, exceto $\mathrm{P} 03$, apresentaram $100 \%$ de acerto em todas as relações simétricas testadas (BA, B-A, CB e $\mathrm{C}-\mathrm{B}$, Figura 3, colunas cinzas). Os percentuais de acerto no teste de emergência das relações simétricas dos participantes P03 e P09 referem-se ao re-teste dessas relações no Protocolo 3. O participante P03 repetiu o Protocolo 2 por não ter atingindo o critério de aprendizagem durante o treino. Pelo mesmo motivo, os participantes P01 e P07 repetiram o Protocolo 1.

Os participantes P01 e P08 apresentaram $100 \%$ de acerto em todos os testes de transitividade e simetria da transitividade. P3, P7 e P9 atingiram percentuais de acerto inferiores a $100 \%$ para algumas das relações de transitividade e simetria da transitividade testadas (Figura 3, colunas pretas). 


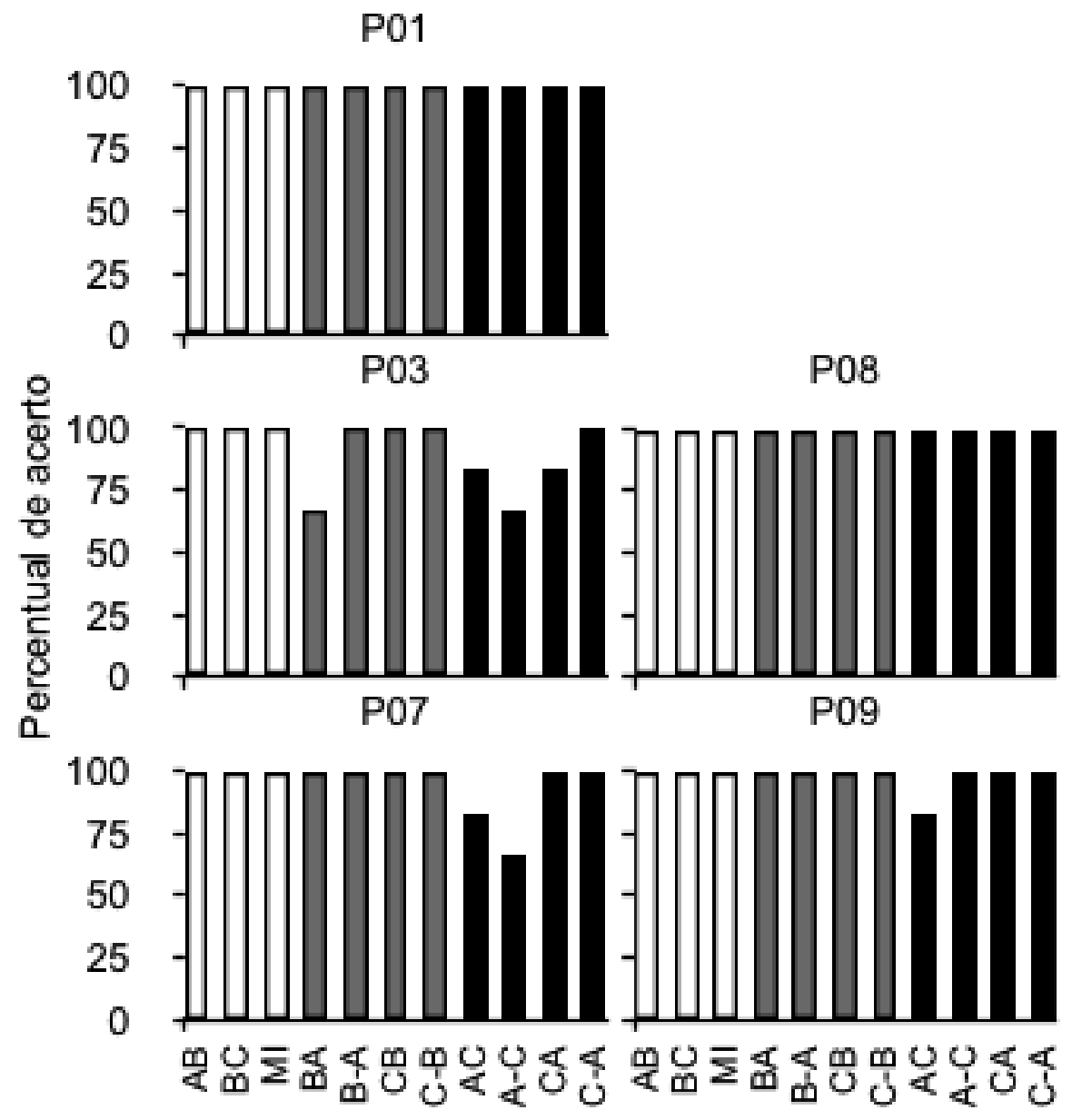

Relações treinadas/testadas

Figura 3. Percentual de acerto no último bloco de cada treino ( $\mathrm{AB}, \mathrm{BC}$ e Misto-MI, colunas brancas), nos testes de simetria (BA, B-A, CB, C-B, colunas cinza), e nos testes de transitividade (AC, A-C, CA e C-A, colunas pretas), para cada participante do Estudo 1. Letras juntas (e.g., AB) indicam que o treino/teste com o procedimento de DSSi. Letras separadas por um traço (e.g., A-B) indicam testes foi com o procedimento de MTS.

\section{Discussão}

Os resultados obtidos no presente estudo mostraram a emergência de relações de equivalência após treino com procedimento de DSSi com duas escolhas, replicando Smeets et al. (2000) e divergindo dos resultados relatados por Moreira e Hanna (2012). O presente estudo sugere que a variabilidade nos testes de transitividade e simetria da transitividade de Moreira e Hanna (2012, Estudo 1) ocorreu, pelo menos em parte, em função da configuração das tentativas (repetição dos mesmos S- em todas as tentativas de treino). Ao reduzir o número de S- de dois para um em cada tentativa, foi possível apresentar diferentes S- entre tentativas.

Os resultados do Estudo 1 do presente trabalho replicam e indicam a generalidade dos resultados de Smeets et al. (2000) ao demostrarem, com dois procedimentos de teste diferentes (DSSI e MTS), a emergência das relações de simetria, transitividade e simetria da transitividade após treino de DSSi.

\section{Estudo 2}

Características não relevantes do estímulo são mencionadas na discussão de estudos como 
possíveis fontes de controle de estímulo não coerente com o programado pelo experimentador (e.g., Sidman, 1992), dificultando a formação sistemática de classes de estímulos equivalentes. Salvo algumas exceções (e.g., McIlvane et al., 1987), a análise de relações de controle diferentes daquelas programadas pelo experimentador, têm sido feitas a posteriori. O Estudo 2 desenvolveu-se em torno da ideia de que seria possível programar um arranjo de estímulos para deliberadamente gerar topografias de controle de estímulos irrelevantes em um procedimento que produziu resultados positivos nos testes. Esse arranjo deveria conter uma característica com probabilidade de reforçamento semelhante à programada para os estímulos positivos, por esta razão chamada de característica redundante. A característica escolhida foi uma mancha adicionada aos estímulos positivos de uma das classes de equivalência potenciais. Escolheu-se uma mancha para ser inserida como aspecto redundante pela facilidade de se adicioná-la aos estímulos já confeccionados, mantendo-se todas as outras características do procedimento iguais às do estudo original. A presença da mancha permitia o acerto sob controle da mesma, isto é, não era necessário que o comportamento do participante ficasse sob o controle da combinação entre os elementos dos estímulos positivos A1B1 e B1C1 também presentes em cada tentativa.

O procedimento selecionado para replicação neste estudo foi o utilizado por Moreira e Hanna (2012, Estudo 2). O Estudo 2 de Moreira e Hanna utilizou uma sequencia de treinos e testes semelhante à descrita no Estudo1 do presente trabalho, porém treinou e testou três classes de equivalência com três estímulos cada em vez de duas. O procedimento escolhido para replicação permitiu incluir o elemento redundante (mancha preta) nos compostos positivos de uma das classes potenciais de equivalência. Se o procedimento do Estudo 1 do presente trabalho fosse utilizado, a presença da mancha em uma das classes tornaria a sua ausência na outra classe uma característica igualmente redundante. A colocação da mancha nos estímulos de apenas uma classe permitiu avaliar se seus efeitos seriam restritos à classe específica ou se as demais classes também seriam afetadas.
O objetivo desse estudo foi avaliar o efeito do ensino de discriminações entre compostos que incluíam um aspecto redundante sobre as classes de estímulos de equivalentes que podem ser formadas.

\section{Método}

\section{Participantes}

Doze universitárias participaram do Estudo 2 (com idades variando entre 19 e 26 anos). Foram concedidos às participantes créditos de atividade complementar pela participação no estudo. Todas as participantes assinaram o Termo de Consentimento Livre e Esclarecido e o projeto foi aprovado pelo Comitê de Ética em Pesquisa com Seres Humanos do Instituto de Ciências Humanas da Universidade de Brasília.

\section{Ambiente Experimental, Equipamento e Estímulos}

Idêntico ao Estudo 1 do presente trabalho.

\section{Procedimento}

O procedimento utilizado foi idêntico ao procedimento utilizado no Estudo 2 de Moreira e Hanna (2012) diferenciando-se apenas pela adição de uma mancha preta aos estímulos compostos A1B1 e B1C1, conforme apresentado na Figura 4 (a mancha não foi inserida nos compostos $\mathrm{A} 2 \mathrm{~B} 2, \mathrm{~B} 2 \mathrm{C} 2, \mathrm{~A} 3 \mathrm{C} 3$ e $\mathrm{B} 3 \mathrm{C} 3$ ). A mancha não foi introduzida em nenhum estímulo durante os testes. O único critério utilizado para o posicionamento da mancha em relação ao estímulo, dentro da janela de resposta, foi estar separada dos elementos do composto, já que ambos eram da mesma cor. A posição relativa da mancha em cada estímulo composto foi a mesma em todas as tentativas (Figura 4).

Devido às semelhanças entre o Estudo 1 do presente trabalho e o Estudo 2 de Moreira e Hanna (2012) é possível compreender o procedimento utilizado aqui descrevendo-se as semelhanças e diferenças entre os dois estudos. Os treinos e testes de cada protocolo descrito no Estudo 1 foram os mesmos, assim como as instruções e a sequência de eventos que compuseram uma tentativa de treino e de teste. No 


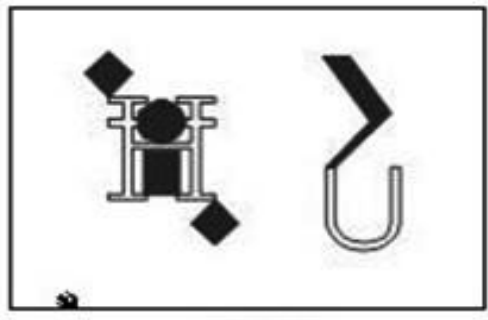

A1B1

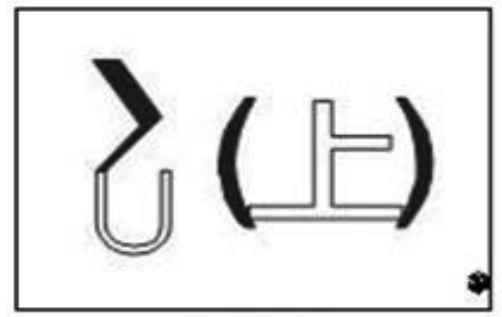

B1C1

Figura 4. Estímulos compostos com a mancha adicionada na parte inferior esquerda de A1B1 e na parte inferior direita de B1C1.

presente estudo, entretanto, foram utilizados três conjuntos de estímulos com três membros em cada conjunto que implicaram na modificação da composição dos blocos dos treinos e testes. Nos Treinos AB e BC foram apresentados respectivamente como estímulos compostos positivos apenas A1B1 e B1C1 nos blocos de 1 a 3, A2B2 e B2C2 nos blocos de 4 a 6 e A3B3 e B3C3 nos blocos de 7 a 9. Nos blocos 10 e 11 todos os estímulos positivos e negativos de seus respectivos treinos foram apresentados alternadamente. No Treino Misto funcionaram como estímulos positivos A1B1, A2B2 e A3B3 nos blocos de 1 a 3 e $\mathrm{B} 1 \mathrm{C} 1, \mathrm{~B} 2 \mathrm{C} 2, \mathrm{~B} 3 \mathrm{C} 3$ nos blocos de 4 a 6 . A partir do bloco 7 , todos os estímulos positivos e negativos foram apresentados alternadamente entre tentativas, sendo a diferença entre os blocos 8 e 9 apenas a ausência de feedback neste último (procedimento de extinção). $\mathrm{O}$ número de $\mathrm{S}$ - presente em cada tentativa aumentou gradualmente de zero a dois ao longo dos blocos. Os S- utilizados foram A1B2, A1B3, A2B1, A2B3, A3B1 e A3B2, para o treino $\mathrm{AB}$ e $\mathrm{B} 1 \mathrm{C} 2, \mathrm{~B} 1 \mathrm{C} 3, \mathrm{~B} 2 \mathrm{C} 1, \mathrm{~B} 2 \mathrm{C} 3$, $\mathrm{B} 2 \mathrm{C} 1$ e $\mathrm{B} 3 \mathrm{C} 2$ para o Treino $\mathrm{BC}$.

Os testes realizados foram os mesmos do Estudo 1 do presente trabalho, mas com três conjuntos de estímulos, sendo cada bloco de teste composto por 18 tentativas totalizando seis apresentações de cada relação testada. Neste estudo a ordem dos testes de transitividade e simetria da transitividade foi alterada, em relação ao Estudo 1 do presente trabalho, para Teste AC, CA, A-C e C-A.

\section{Resultados}

As porcentagens de acertos nos treinos e testes são apresentadas na Figura 5. As primeiras três colunas de cada gráfico, na cor branca, mostram os percentuais de acerto de cada participante no último bloco de tentativas de cada treino. Todos os participantes atingiram $100 \%$ de acerto no último bloco dos Treinos AB, BC e Misto.

Os resultados dos testes de simetria (BA, B-A, CB, C-B) são apresentados nas colunas cinzas e os desempenhos nos testes de transitividade e simetria da transitividade nas colunas pretas da Figura 5. Todos os participantes, exceto $\mathrm{P} 04$, atingiram $100 \%$ de acerto, ou valores próximos, em todos os testes de emergência das relações simétricas. No entanto, apenas os participantes P13, P16 e P18 apresentaram a emergência de todas as relações de transitividade e simetria da transitividade. Os demais participantes apresentaram percentuais de acerto baixos e variados nos testes de transitividade e simetria da transitividade e não se verificou diferenças sistemáticas em função do procedimento de teste utilizado (DSSi ou MTS).

A Figura 6 mostra o número de acertos por relação para cada participante nos testes de transitividade e simetria da transitividade. Não houve diferenças sistemáticas entre os desempenhos nos testes com o Conjunto 1 (que possuíam a mancha no treino) e aqueles sem a mancha (Conjuntos 2 e 3 ).

Cinco dos doze participantes $(41,7 \%$ - P 01 , P09, P15, P16 e P18) responderam de acordo 
$\begin{array}{lll}\text { P01 P02 } & \text { P04 }\end{array}$

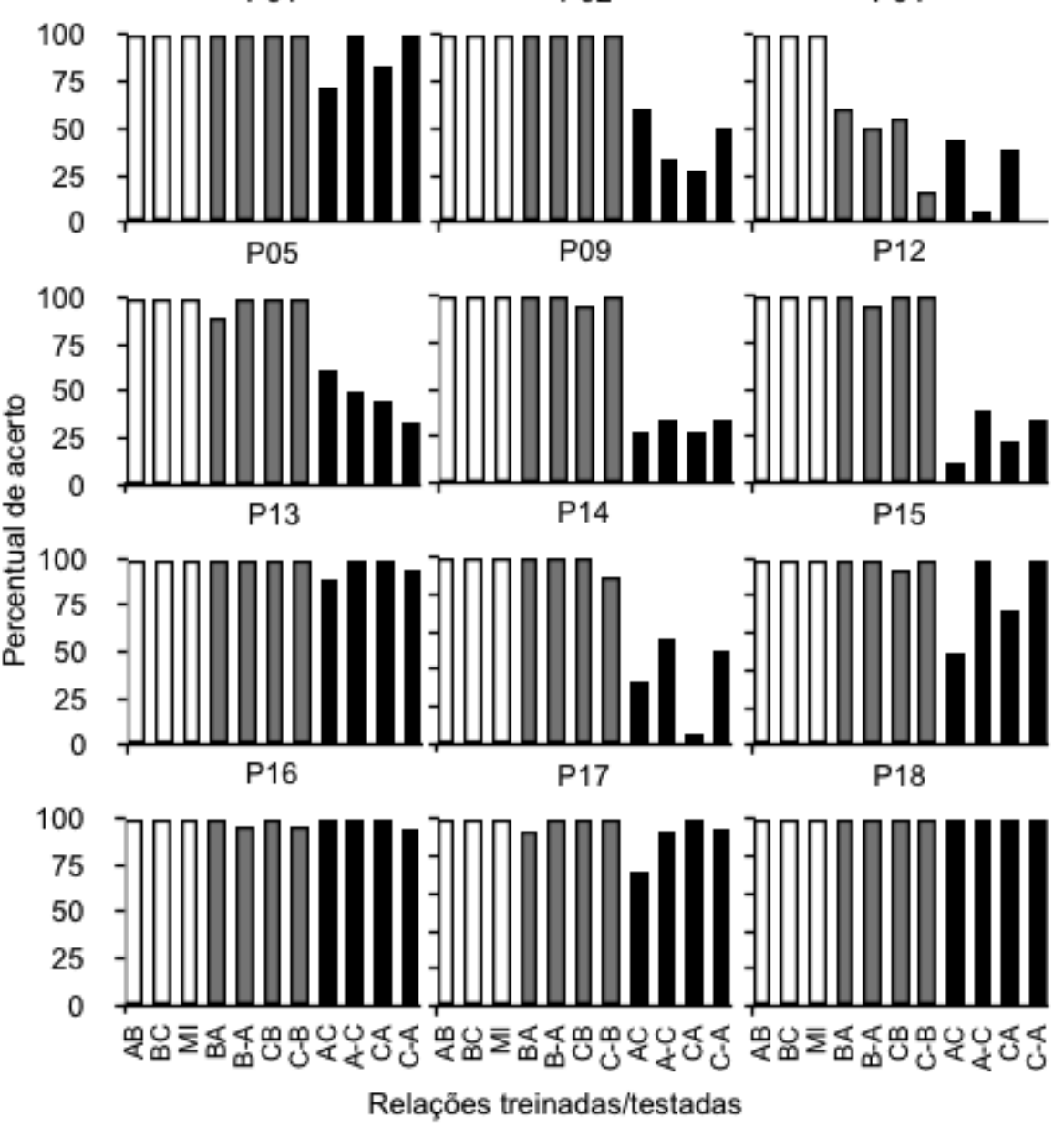

Figura 5. Percentual de acerto no último bloco de cada treino $(\mathrm{AB}, \mathrm{BC}$ e Misto-MI, colunas brancas), nos testes de simetria (BA, B-A, CB, C-B, colunas cinzas), e nos testes de transitividade (AC, A-C, CA e C-A, colunas pretas), para cada participante do Estudo 2. Letras juntas (e.g., AB) indicam treino/ teste com o procedimento de DSSi. Letras separadas por um traço (e.g., A-B) indicam testes com o procedimento de MTS.

com as relações definidas pelo experimentador em todas ou em cinco das seis tentativas com estímulos do Conjunto 1 . Seis dos doze participantes $(50 \%$ - P01, P05, P13, P16, P17 e P18) responderam de acordo com as relações programadas em todas ou em cinco das seis tentativas com estímulos do Conjunto 2. Para o Conjunto 3, quatro dos doze participantes $(33,3 \%$ - P13, $\mathrm{P} 16, \mathrm{P} 17$ e P18) responderam de acordo com as relações programadas em todas ou em cinco das seis tentativas relativas a esse conjunto de estímulos.

Quando comparado o número de erros cometidos em cada procedimento de teste, observa-se que cinco participantes (P01, P09, P12, $\mathrm{P} 13$, e P15) apresentaram mais erros nos testes com DSSi, três participantes (P04, P05 e P17) apresentaram mais erros nos testes com MTS e quatro participantes (P02, P14, P16 e P18) apresentaram o mesmo número de erros em ambos os procedimentos. P16 e P18 cometeram zero erro. Somados os erros de todos os participantes, observa-se um total de 164 erros durante os testes com DSSi e 155 erros durante os testes com MTS (cada teste, independentemente do procedimento, foi constituído por um bloco com 18 tentativas). O maior número de erros ocorreu durante os testes com DSSi do Conjunto 3. A análise das escolhas dos participantes revelou o desenvolvimento de relações de controle estímulo-estímulo diferente entre eles e diferentes daquelas programadas pelo experimentador. Por exemplo, P09 clicou sobre A2C3 nas seis tentativas nas quais este $S$ - estava presente. Não se observou escolhas sistemáticas de compostos negativos entre participantes. 


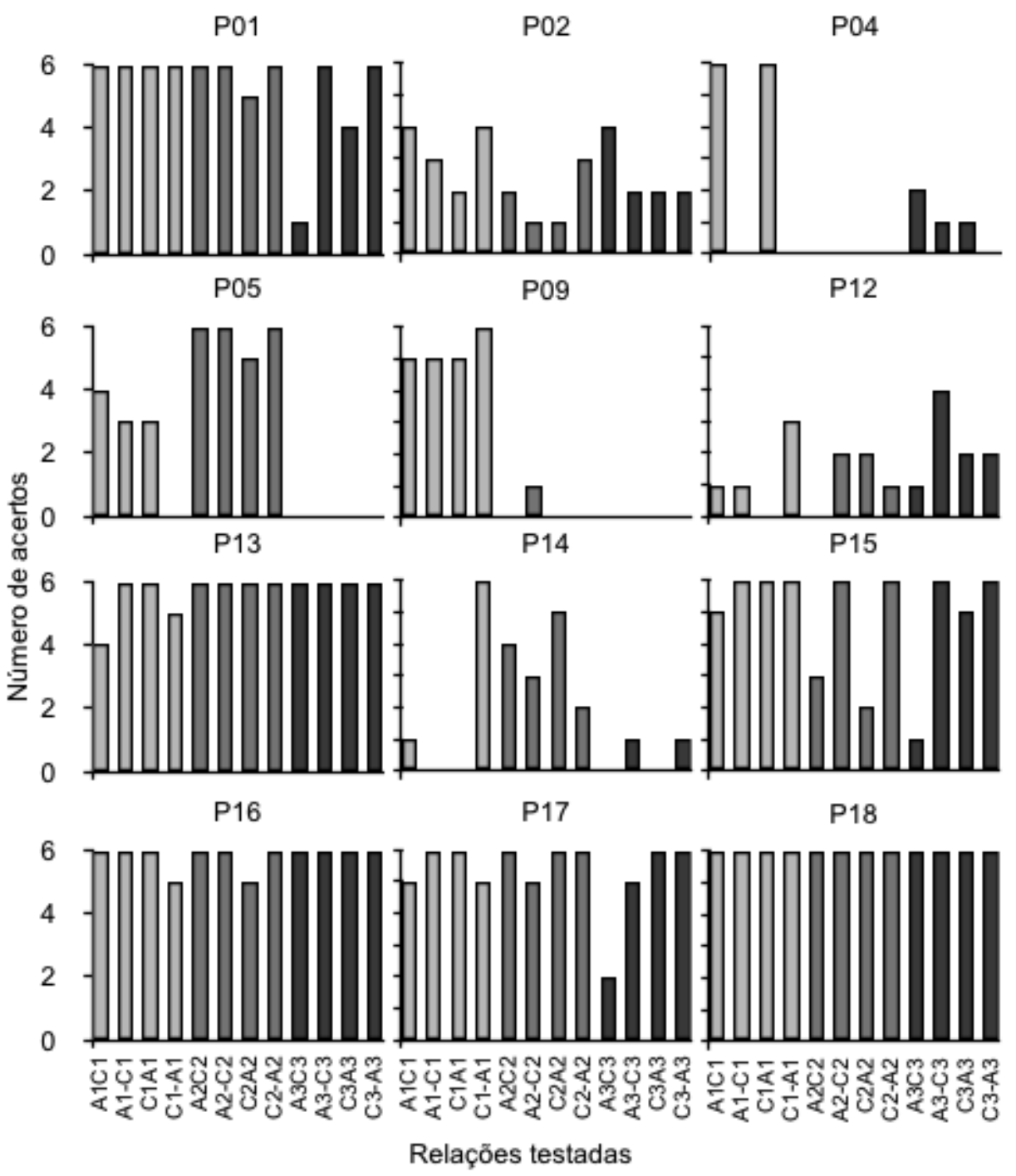

Figura 6. Número de acertos por conjunto nos testes de emergência das relações de transitividade e simetria da transitividade do Estudo 2. Letras juntas (e.g., A1C1) indicam que o teste foi realizado utilizando-se o procedimento de DSSi. Letras separadas por um traço (e.g., A1-C1) indicam que o teste foi realizado utilizando-se o procedimento de MTS.

\section{Discussão}

No Estudo 2 de Moreira e Hanna (2012), cinco participantes foram expostos a um treino de DSSi com estímulos compostos e procedimento semelhante ao utilizado no presente estudo. A emergência das relações de simetria, transitividade e simetria da transitividade foi observada para todos os participantes tanto com um procedimento de DSSi quanto com um procedimento de MTS. No presente estudo, que incluiu uma mancha nos estímulos de uma das classes ensinadas (A1B1 e B1C1), apenas três participantes (P13, P16 e P18) apresentaram a emergência de todas as relações de transitividade e simetria da transitividade. Esses resultados, quando comparados aos resultados do Estudo 2 de Moreira e Hanna, sugerem fortemente que a característica redundante presente no ensino das relações foi eficaz na produção relações de controle estímulo-estímulo diferentes daquelas programadas pelo experimentador e, consequentemente, em prevenir a formação de classes de equivalência de estímulos (Dube \& McIlvane, 1996).

À exceção de $\mathrm{P} 4$, todos os participantes demonstraram a emergência das relações de simetria, sugerindo que a mancha não exerceu efeito na emergência dessas relações. Esse resultado 
replica os resultados relatados por Moreira e Hanna (2012) em seu Estudo 2. Uma vez que os elementos dos compostos $\mathrm{S}+$ estavam presentes nas tentativas dos testes de simetria, apenas em posição invertida, é possível que eles tenham adquirido algum controle discriminativo e não apenas a mancha. Testes com máscara (Mcllvane et al., 1987) poderiam ser utilizados em estudos futuros para auxiliar na compreensão do controle comportamental pelos elementos dos estímulos quando o $\mathrm{S}+$, o $\mathrm{S}$ - ou parte deles é coberto com e sem a mancha presente.

Uma explicação alternativa para os altos escores nos testes de simetria é considerá-los como "falsos positivos", descrevendo-os como evidência de generalização de estímulos (Smeets et al., 2000). A semelhança física dos estímulos compostos utilizados nos teses de simetria com procedimento de DSSi em relação aos estímulos compostos utilizados nos treinos (espelhados) pode ter sido suficiente para manter o controle comportamento pela nova configuração. No entanto, ao se descrever os resultados positivos nos testes de simetria como generalização de estímulos, ainda se pode questionar se a mesma descrição poderia ser aplicada aos testes de simetria com o procedimento de MTS, no qual os elementos que formavam o composto nos treinos foram separados e apresentados ora como estímulo modelo, ora como estímulo de comparação (e.g., A1 e B1)?

Embora a mancha tenha sido inserida apenas nos estímulos compostos do Conjunto 1 (A1B1 e B1C1), observou-se variabilidade na emergência das relações de transitividade e simetria da transitividade dos três conjuntos de estímulos. Uma possível explicação para o efeito generalizado da mancha pode ser baseada na sugestão de Sidman (2000) de que respostas e reforços podem também fazer parte de classes de equivalência. De acordo com Sidman, se respostas e reforços fazem parte de relações de equivalência, respostas e reforços comuns a duas ou mais classes poderiam gerar uma grande classe de equivalência composta por todos os elementos (modelos, comparações, respostas e reforços). Quando esta grande e única classe é formada, o desempenho em testes de relações emergentes das classes menores será inconsistente e os resultados negativos. Alguns indivíduos, entretanto, podem discriminar entre classes mesmo com respostas e reforço comuns, como também aponta Sidman e diversos resultados da literatura (e.g., Markham \& Dougher, 1993; Smeets et al., 2000; Stromer \& Stromer, 1990). Para resolver essa inconsistência, Sidman (2000) sugere que "as demandas das contingências, no entanto, devem fazer com que os elementos respostas e reforços eventualmente saiam daquela classe, tornando possível a formação das classes menores, $\mathrm{A} 1 \mathrm{~B} 1 \mathrm{Cl}$ e A2B2C2" (p. 132).

\section{Discussão Geral}

No presente trabalho dois estudos investigaram o efeito de características redundantes das configurações das tentativas de treino na formação de classes de equivalência de estímulos utilizando-se um procedimento de treino com DDSi com estímulos compostos. Há ampla evidência na literatura de que as discriminações programadas pelo experimentador nem sempre são as discriminações aprendidas pelo sujeito experimental (e.g., Dube et al., 2010; Dube \& McIlvane, 1996; Lashley, 1938; Ray, 1969; Reynolds, 1961; Touchette, 1969). Esse fenômeno tem sido referido na literatura como controle de estímulos restrito (Dube \& Mcllvane, 1997) ou superseletividade de estímulos (Lovaas, Koegel, \& Schreibman, 1979).

O estabelecimento de controles de estímulos diferentes daqueles programados pelo experimentador durante tarefas de treino discriminativo é por si só um problema de pesquisa legítimo quando o ensino de discriminações é o fenômeno em questão (e.g., Dube \& McIlvane, 1997). Além disso, o estabelecimento de topografias de controle de estímulos incongruentes com as programadas pelo experimentador está diretamente relacionado a falhas na emergência de classes de equivalência de estímulos (Dube \& McIlvane, 1996). Os resultados obtidos no presente trabalho expandem o corpo de evidências de que configurações das tentativas de treino e/ ou características dos estímulos podem gerar falsos-positivos nos treinos discriminativos e 
falhas na emergência das relações de transitividade e simetria da transitividade (cf., Dube \& McIlvane, 1996) ao demonstrar a ocorrência deste fenômeno em treinos discriminativos utilizando um procedimento de DSSi.

Os resultados do Estudo 1 do presente trabalho, quando comparados aos resultados do Estudo 1 de Moreira e Hanna (2012), exemplificam um caso de identificação do desenvolvimento de topografias de controle de estímulos incongruentes a posteriori, como é o caso comum na literatura - isto é, as topografias são inferidas apenas após a constatação de falhas na formação das classes de equivalência ou identificadas apenas após a realização de um novo estudo ou experimento. Neste sentido, o Estudo 2 do presente trabalho apresenta uma segunda contribuição potencialmente relevante para o desenvolvimento de procedimentos que previnam ou auxiliem a identificar o desenvolvimento de topografias de controle de estímulos incongruentes durante tarefas de treino discriminativo: a inserção da característica redundante (a mancha) em parte dos estímulos.

A inserção da característica redundante em apenas um conjunto de estímulos, no Estudo 2, produziu falhas na emergência das relações de transitividade e simetria da transitividade dos demais conjuntos de estímulos. Não é possível identificar, no presente trabalho, quais variáveis teriam sido responsáveis por esses resultados e a proposta de Sidman (2000) descrita anteriormente precisaria ser testada empiricamente neste caso. Para identificar tais variáveis, talvez uma primeira manipulação pudesse ser a retirada das tentativas de treino referentes ao Conjunto 1 , mantendo-se as tentativas relativas ao Conjunto 1 nos testes. Desta forma, poder-se-ia avaliar se as falhas na emergência das relações de transitividade e simetria da transitividade, nas três classes, são função somente da manipulação realizada no treino das relações do Conjunto 1 , ou se também são função da presença, nos testes, de tentativas com "estímulos não-relacionados".

No Estudo 2, a mesma resposta era requerida e as mesmas consequências apresentadas para os três conjuntos de estímulos. A elaboração de um procedimento que contemplasse a manipula- ção realizada no Estudo 2, mas que também previsse respostas e consequências diferentes para as relações treinadas de cada conjunto, parece promissora não só para se verificar se os efeitos da manipulação do Estudo 2 fica restrito à classe na qual se inseriu a mancha, mas também para testar a hipótese de Sidman (2000) citada anteriormente (e.g., Dube \& McIlvane, 1995; Dube, McIlvane, Mackay, \& Stoddard, 1987; Garcia \& Benjumea, 2006; Urcuioli, Lionello-DeNolf, Michalek, \& Vasconcelos, 2006; Urcuioli \& Vasconcelos, 2008).

Os resultados do presente trabalho corroboram os resultados de Moreira e Hanna (2012) ao demonstrarem desempenhos similares dos participantes nos testes com MTS e nos testes com DSSi após treino com DSSi com estímulos compostos. Tais resultados fornecem suporte empírico para a noção de que elementos de um estímulo composto podem ser separados e recombinados sem que haja ruptura no controle discriminativo (Debert et al., 2009; Debert, Matos, \& Andery, 2006; Debert et al., 2007; Markham \& Dougher, 1993).

Conforme apontam Debert et al. (2006), não é possível identificar as funções condicionais e discriminativas dos estímulos em procedimentos de treino de discriminações simples com estímulos compostos. Se essa proposição se provar verdadeira, será necessário rever as propriedades definidoras de uma classe de equivalência de estímulos. A propriedade simétrica, por exemplo, é definida com base na inversão das funções dos estímulos (discriminativa e condicional). No presente trabalho, assim como em Moreira e Hanna (2012), a emergência das chamadas relações simétricas pouco ou nada foi afetada pelas redundâncias presentes durante os treinos discriminativos.

Os dois estudos são evidências adicionais de que o estabelecimento de controle por aspectos não programados ou não desejados do ambiente podem ter efeito deletério na aprendizagem discriminativa e relacional. Em situação natural, a identificação e controle de todas as possíveis fontes não é tarefa fácil, se é que é possível. Ainda assim, o conhecimento mais amplo sobre as condições que favorecem topografias de estímu- 
los desejados é importante e necessário para a geração de tecnologia de ensino eficiente.

\section{Referências}

Carpentier, F., Smeets, P. \& Barnes-Holmes, D. (2000). Matching compound samples with unitary comparisons: Derived stimulus relations in adults and children. The Psychological Record, 50, 671-685. Retirado de http://opensiuc.lib.siu.edu/cgi/viewcontent. cgi? article $=1440 \&$ context $=$ tpr

Debert, P., Huziwara, E. M., Faggiani, R. B., de Mathis, M. E. S., \& McIlvane, W. J. (2009). Emergent conditional relations in a go/no-go procedure: Figure-ground and stimulus-position compound relations. Journal of Experimental Analysis of Behavior, 92, 233-243. doi:10.1901/ jeab.2009.92-233

Debert, P., Matos, M. A., \& Andery, M. A. P. A. (2006). Discriminação condicional: Definições, procedimentos e dados recentes. Revista Brasileira de Análise do Comportamento, 2, 37-52. Retirado de http://www.periodicos.ufpa.br/index.php/rebac/article/view/801/1111

Debert, P., Matos, M. A., \& McIlvane, W. (2007). Conditional relations with compound abstract stimuli using a go/no-go procedure. Journal of Experimental Analysis of Behavior, 87, 89-96. doi:10.1901/jeab.2007.46-05

Dube, W. V., Dickson, C. A., Balsamo, L. M., O’Donnell, K. L., Tomanari, G. Y., Farren, K. M., ...McIlvane, W. J. (2010). Observing behavior and atypically restricted stimulus control. Journal of the Experimental Analysis of Behavior, 94, 297-313. doi:10.1901/ jeab.2010.94-297

Dube, W. V., \& McIlvane, W. J. (1995). Stimulusreinforcer relations and emergent matching to sample. The Psychological Record, 45, 591-612.

Dube, W. V., \& McIlvane, W. J. (1996). Some implications of a stimulus control topography analysis for emergent stimulus classes. In T. R. Zentall \& P. M. Smeets (Eds.), Stimulus class formation in humans and animals (pp. 197-218). Amsterdam, North Holland: Elsevier.

Dube, W. V., \& McIlvane, W. J. (1997). Reinforcer frequency and restricted stimulus control. Journal of Experimental Analysis of Behavior, 68, 303-316. doi:10.1901/jeab.1997.68-303
Dube, W. V., McIlvane, W. J., Mackay, H. A., \& Stoddard, L. T. (1987). Stimulus class membership established via stimulus-reinforcer relations. Journal of the Experimental Analysis of Behavior, 47, 159-175. doi:10.1901/ jeab.1987.47-159

Garcia, A., \& Benjumea, S. (2006). The emergence of symmetry in a conditional discrimination task using different responses as propioceptive samples in pigeons. Journal of the Experimental Analysis of Behavior, 86, 65-80. doi:10.1901/ jeab.2006.67-04

Grisante, P. C., Galesi, F. L., Sabino, N. M., Debert, P., Arntzen, E., \& McIlvane, W. J. (2013). Go/ no-go procedure with compound stimuli: Effects of training structure on the emergence of equivalence classes. The Psychological Record, 63, 63-72.

Groskreutz, N. C., Karsina, A., Miguel, C. F., \& Groskreutz, M. P. (2010). Using complex auditory-visual samples to produce emergent relations in children with autism. Journal of Applied Behavior Analysis, 43, 131-136. doi:10.1901/ jaba.2010.43-131

Guerrero, M., Alós, F. J., \& Moriana, J. A. (2015). Emergent relations with compound stimuli in conditional and simple discriminations: An experimental application in children. The Psychological Record, 65, 475-486. doi:10.1007/ s40732-015-0123-6

Lashley, K. S. (1938). The mechanism of vision: XV. Preliminary studies of the rat's capacity for detail vision. Journal of General Psychology, $18,123-193$.

Lovaas, O. I., Koegel, R. L., \& Schreibman, L. (1979). Stimulus overselectivity in autism: A review of research. Psychological Bulletin, 86, $1236-1254$

Maguire, R. W., Stromer, R., Mackay, H. A., \& Demis, C. A. (1994). Matching to complex samples and stimulus class formation in adults with autism and young children. Journal of Autism and Developmental Disorders, 24, 753772 .

McIlvane, W. J., Kledaras, J. B., Munson, L. C., King, K. A., De Rose, J. C., \& Stoddard, L. T. (1987). Controlling relations in conditional discrimination and matching by exclusion. Journal of the Experimental Analysis of Behavior, 48, 187-208. doi:10.1901/jeab.1987.48-187 
Markham, M. \& Dougher, M. J. (1993). Compound stimuli in emergent stimulus relations: Extending the scope of stimulus equivalence. Journal of the Experimental Analysis of Behavior, 60, 529-542. doi:10.1901/jeab.1993.60-529

Moreira, M. B., \& Hanna, E. S. (2012). Emergência de classes de equivalência após separação e recombinação dos estímulos compostos utilizados no treino. Revista Brasileira de Análise do Comportamento, 8, 59-80. Retirado de http:// www.periodicos.ufpa.br/index.php/rebac/article/view/1922/2345

Nalini, L. E. (2002). Determinação empírica da nomeabilidade de estímulos: Implicações para o estudo da relação de nomeação (Tese de doutorado, Universidade de Brasília, DF, Brasil).

Pérez-González, L. A., \& Alonso-Álvarez, B. (2008). Common control by compound samples in conditional discriminations. Journal of the Experimental Analysis of Behavior, 90, 81-101. doi:10.1901/jeab.2008.90-81

Ray, B. A. (1969). Selective attention: The effects of combining stimuli which control incompatible behavior. Journal of the Experimental Analysis of Behavior, 12, 539-550. doi:10.1901/ jeab.1969.12-539

Reynolds, G. (1961). Attention in the pigeon. Journal of the Experimental Analysis of Behavior, 4, 203-208. doi:10.1901/jeab.1976.26-207

Ribeiro, D. M., Miguel, C. F., \& Goyos, C. (2015). The effects of listener training on discriminative control by elements of compound stimuli in children with disabilities. Journal of the Experimental Analysis of Behavior, 104, 48-62. doi:10.1002/jeab.161

Sidman, M. (1992). Adventitious control by the location of comparison stimuli in conditional discriminations. Journal of the Experimental Analysis of Behavior, 58, 173-182.

Sidman, M. (2000). Equivalence relations and the reinforcement contingency. Journal of the Experimental Analysis of Behavior, 74, 127146. doi:10.1901/jeab.1992.58-173

Sidman, M., Rauzin, R., Lazar, R., Cunningham, S., Tailby, W., \& Carrigan, P. A. (1982). Search for symmetry in the conditional discriminations of rhesus monkeys, baboons, and children. Journal of the Experimental Analysis of Behavior, 37, 23-44. doi:10.1901/jeab.1982.37-23
Sidman, M., \& Tailby, W. (1982). Conditional discrimination vs. matching to sample: An expansion of the testing paradigm. Journal of the Experimental Analysis of Behavior, 37, 5-22. doi:10.1901/jeab.1982.37-5

Smeets, P. M., Barnes-Holmes, D., \& Cullinan, V. (2000). Establishing equivalence classes with match-to-sample format and simultaneous-discrimination format conditional discrimination tasks. The Psychological Record, 50, 721-744. Retirado de http://opensiuc.lib.siu.edu/cgi/viewcontent.cgi? article $=1443 \&$ context $=$ tpr

Stromer, R., McIlvane, W., \& Serna, R. (1993). Complex stimulus control and equivalence. The Psychological Record, 43, 585-598.

Stromer, R., \& Stromer, J. (1990). The formation of arbitrary stimulus classes in matching to complex samples. The Psychological Record, 40, 51-66.

Touchette, P. E. (1969). Tilted lines as complex stimuli. Journal of the Experimental Analysis of Behavior, 12, 211-214. doi:10.1901/ jeab.1969.12-211

Urcuioli, P. J., Lionello-DeNolf, K., Michalek, S., \& Vasconcelos, M. (2006). Some tests of response membership in acquired equivalence classes. Journal of the Experimental Analysis of Behavior, 86, 81-107. doi:10.1901/ jeab.2006.52-05

Urcuioli, P. J., \& Vasconcelos, M. (2008). Effects of within-class differences in sample responding on acquired sample equivalence. Journal of the Experimental Analysis of Behavior, 89, 341358. doi:10.1901/jeab.2008-89-341 